\title{
Modelando o conhecimento algébrico do estudante através de Redes Bayesianas Dinâmicas
}

\author{
Henrique M. Seffrin ${ }^{1}$, Patricia Jaques ${ }^{1}$ \\ ${ }^{1}$ Programa Interdisciplinar de Pós-Graduação em Computação Aplicada (PIPCA) \\ Universidade do Vale do Rio dos Sinos (UNISINOS) \\ hseffrin@outlook.com, patricia.jaques@gmail.com
}

\begin{abstract}
Bayesian Networks (BN) are widely used in the literature to perform inference on uncertain data, including to diagnostic students' knowledge in Intelligent Tutoring Systems. One of the main challenges in modeling this type of component is to infer the students' knowledge degree in each knowledge unit and relate them correctly. This paper presents a structure of $B N$ to model the algebraic knowledge of students for $1^{\text {st }}$ degree equations, which relates the main operations with their properties and concepts. In order to validate it, we conducted interviews with math teachers, who examined this structure and expressed their opinion. These interviews also allowed to get the main mistakes made by students in the classroom, which will allow to include inference to misconceptions in the proposed $B N$.
\end{abstract}

Resumo. Redes Bayesianas (RB) são muito utilizadas na literatura para realizar inferência sobre dados incertos, inclusive para diagnóstico do conhecimento dos estudantes em Sistemas Tutores Inteligentes. Um dos desafios encontrados ao modelar este tipo de componente é inferir o grau de conhecimento do estudante em cada unidade de conhecimento e relacioná-las corretamente. Este artigo apresenta uma estrutura de RB que modela o conhecimento algébrico dos aprendizes para equações de $1^{\circ}$ grau, relacionando as principais operações, com suas propriedades e respectivos conceitos. De modo a avalia-la, foram conduzidas entrevistas com professores da área de matemática, que analisaram esta estrutura e emitiram um parecer. As entrevistas também permitiram levantar os principais erros cometidos pelos alunos em sala de aula, que serão futuramente incluídos na RB proposta.

\section{Introdução}

A álgebra é uma área da matemática em que os alunos apresentam grandes dificuldades. Isto se deve principalmente ao fato que este conteúdo apresenta uma quebra de paradigmas em relação à aritmética tradicional, que era ensinada até então nas escolas. Ela exige que o estudante desenvolva o raciocínio algébrico, isto é, que ele seja capaz de interpretar situações e abstrair os seus conceitos de modo a descobrir os valores que são desconhecidos (variáveis ou incógnitas). Softwares educacionais podem auxiliar o estudante na compreensão destes novos conceitos. Nesta categoria de software, destacam-se os Sistemas Tutores Inteligentes.

Sistemas Tutores Inteligentes (STI) são softwares educacionais voltados para a aprendizagem de conteúdos. São caracterizados por empregarem técnicas de Inteligência 
Artificial em sua arquitetura, para resolver questões relacionadas ao seu domínio, e de se adaptarem às características de cada estudante [Woolf 2008]. Isto permite um ensino mais focado no conhecimento de cada aluno, assim como permite que o processo de ensino esteja de acordo com o ritmo de cada um.

Esta capacidade adaptativa dos STIs é provida, principalmente, pelo componente Modelo de Aluno. Este é constituído de uma base de dados, que contém o perfil de cada estudante do sistema, como nome, idade, sexo, turma, escola; características relativas ao seu aprendizado, como o seu estilo de aprendizado, seu histórico de exercícios e atividades, e o seu conhecimento; e por fim as características afetivas deste aluno, como estado emocional e motivação.

Modelos de Aluno também possuem um componente para avaliar o conhecimento de cada aluno, o que permite a característica adaptativa do sistema. Conforme apresentado em [Vanlehn 2006], as etapas de um exercício são uma das poucas evidências de conhecimento que o estudante pode mostrar ao sistema. Desta forma, este processo de avaliação de conhecimento ocorre conforme o aluno interage com o STI, resolvendo os exercícios propostos, passo a passo.

A implementação de um Modelo de Aluno pode ser um desafio, uma vez que este tipo de inferência é dependente de domínio, e cada área de conhecimento possui sua estruturação própria. Deve-se identificar as suas principais unidades de conhecimento, definir uma relação entre elas, e o nível de importância de cada relação. No domínio algébrico, isto é observado através das relações entre as operações e os seus principais conceitos, como incógnitas e igualdade entre os lados da equação.

$\mathrm{Na}$ literatura são encontrados diversos trabalhos relacionados à inferência de conhecimento dos estudantes que empregam redes Bayesianas. No domínio algébrico são encontrados os trabalhos de [Millán et al. 2013], [Duijnhoven 2003] e [Seffrin et al. 2013]. O primeiro modela uma rede Bayesiana que descreve uma relação entre os conceitos algébricos para operações de $1^{\circ}$ grau. $\mathrm{O}$ segundo descreve um modelo mais complexo, relacionando as principais operações com suas respectivas propriedades e falsas concepções. Já o terceiro apresenta uma modelagem mais simples, estabelecendo relações, apenas, entre as operações de $1^{\circ}$ grau.

Estes trabalhos possuem duas lacunas em comum. Eles obtém as informações a partir de itens de avaliação, e, por isso, são restritos aos exercícios modelados na rede. Isso significa que para que o STI possa aplicar novos exercícios, a rede deve ser estendida, o que igualmente traz maior complexidade à rede. Além disso, eles modelam apenas os conceitos ou as habilidades algébricas dos estudantes e não consideram as relações entre esses. No entanto, na álgebra observa-se que conceitos e operações estão diretamente relacionados [Matz 1982]. Esse é o caso da operação inversa, que está diretamente ligada ao conceito de "equilíbrio entre os lados da equação", ou seja, de modo a "mover um termo para o outro lado da equação" é necessário somar/subtrair ou multiplicar/dividir em ambos os lados desta equação. Desta forma, é desejável que um modelo de inferência seja capaz de identificar estes conceitos e suas relações com as habilidades (operações, no caso da álgebra).

A proposta deste trabalho é definir um modelo de inferência, empregando redes Bayesianas dinâmicas, que seja capaz de tratar estas limitações. O modelo proposto 
deve ser capaz de identificar os principais conceitos algébricos que o aluno conhece, e, desta forma, inferir se ele é capaz de aplicar determinada operação, ou caso ele não seja, deve identificar que conceito relacionado ele não compreende. Para isto, é necessária a elaboração de uma estrutura que relacione cada operação algébrica com os seus principais conceitos e falsas concepções. Também busca-se desenvolver uma rede que seja independente dos exercícios para maior flexibilidade do tutor. Entrevistas a professores permitiram avaliar qualitativamente a rede proposta.

Estando o modelo finalizado, ele será incorporado ao modelo de aluno do STI PAT2Math ${ }^{1}$ (Personal Affective Tutor to Math). Neste tutor, o modelo irá atuar na identificação do conhecimento algébrico do estudante, utilizando como evidência as habilidades dos alunos que foram identificadas pelo componente cognitivo do sistema (sistema especialista), durante a resolução das tarefas.

Este trabalho está organizado como segue. A seção 2 apresenta a fundamentação sobre redes Bayesianas e redes Bayesianas Dinâmicas, que é a estrutura adotada para a implementação do modelo de inferência proposto. Na seção 3, são listados os trabalhos relacionados à avaliação de conhecimento do aluno. O trabalho proposto neste artigo é descrito na seção 4, e a sua avaliação, ou seja, a entrevista realizada com os professores, é apresentada na seção 5. Finalizando, as conclusões e trabalhos futuros são apresentados na seção 6.

\section{Redes Bayesianas}

Redes Bayesianas (RBs) são modelos probabilísticos utilizados na inferência de dados incertos [Russell and Norvig 2002]. São representadas através de grafos direcionados acíclicos (Directed Acyclic Graphs - DAG), que representam tanto as dependências, como as independências condicionais e incondicionais entre as variáveis [Pearl 1988].

Cada variável é representada por um vértice (ou nodo) no grafo, e os arcos do grafo representam as relações entre elas. Portanto, se um nodo $Y$ recebe um arco de um nodo $X$, ele tem a sua probabilidade definida pela probabilidade condicional $P(Y \mid X)$. Da mesma forma, se nenhuma variável estende um arco para $X$, este possui apenas as suas probabilidades independentes, ou a priori, que são definidas apenas por $P(X)$ [Pearl 1988].

Estas probabilidades definem as chances que uma variável possui de assumir determinado valor ou estado. Cada variável possui um conjunto finito de estados que pode assumir. No geral, as redes trabalham apenas com nodos binários, com 2 estados. Além destes, cada nodo possui uma tabela de probabilidades condicionais, que define os valores que este nodo irá assumir, dado o valor dos nodos condicionados a ele. Desta forma, através de uma RB é possível obter qualquer informação, referente a qualquer variável da rede [Pearl 1988]. Segundo [Russell and Norvig 2002], RBs são uma forma compacta de representar a distribuição conjunta de probabilidade de um conjunto de variáveis.

O processo de inferência nas RBs ocorre por meio da apresentação de evidências, que são certezas, fatos que podem ser observados no ambiente modelado pela RB. Nas RBs, as variáveis, que recebem as evidências, tem a probabilidade de ocorrência de um de seus estados alterado para $100 \%$. Uma vez que todas as evidências foram informadas à rede ocorre a propagação, ou seja, os valores das probabilidades das

\footnotetext{
${ }^{1}$ http: //pat2math. unisinos.br
} 
outras variáveis são ajustados de acordo com estas novas informações das evidências [Russell and Norvig 2002].

Conforme [Russell and Norvig 2002], duas importantes formas de inferência em RBs são a predição e o diagnóstico. Para exemplificar estes métodos de inferência considere um modelo causal, no qual a rede é modelada de modo a representar uma estrutura de causa e efeito, ou seja, a "causa" tem influência direta na probabilidade de ocorrência do "efeito". Na predição, as evidências são apresentadas nas causas, e deseja-se observar quais as chances de ocorrência de determinados efeitos. Já para o diagnóstico, as evidências são apresentadas aos efeitos e deseja-se observar as possíveis causas para eles. Por exemplo, dado que um paciente apresenta os sintomas $s_{1}$ e $s_{2}$, quais as possíveis doenças que este paciente desenvolveu.

\subsection{Redes Bayesianas Dinâmicas}

Redes Bayesianas Dinâmicas (RBDs), ou redes Bayesianas Temporais, são um tipo especial de RBs que trabalham com inferências ao longo do tempo. Elas utilizam o conceito de time-slices ou instantes de tempo. Além das probabilidades utilizadas pelas RBs, RBDs definem mais três tipos de probabilidades, que estão relacionadas ao tempo [Russell and Norvig 2002]: a distribuição do estado inicial, $P\left(X_{0}\right)$, as probabilidades de transição entre os instantes de tempo, $P\left(X_{t+1} \mid X_{t}\right)$, e os nodo-sensores, $P\left(E_{t} \mid X_{t}\right)$, os nodos que receberão as evidências $E$ do instante de tempo $t$.

O processo de inferência em RBDs consiste na apresentação de evidências aos nodos-sensores. As informações de um time-slice serão propagadas para o próximo através dos arcos temporais. Estes definem a influência que um nodo, no tempo $t$, irá exercer sobre si mesmo em um tempo $t+1$. Uma RB pode ser convertida em um RBD através de um processo chamado unrolling, que consiste em criar finitas cópias da RB original, uma para cada time-slice da RBD. Estas cópias estarão unidas uma às outras na RBD através dos arcos temporais [Russell and Norvig 2002].

\section{Trabalhos Relacionados}

RBs e RBDs tem sido empregadas para avaliação do conhecimento dos aprendizes em STIs. O Knowledge Tracing [Corbett and Anderson 1994], principal trabalho nesta área, avalia o conhecimento algébrico do aluno através de cálculos de probabilidade. Ele é baseado na teoria cognitiva $\mathrm{ACT}^{*}$, que define o conhecimento como um conjunto de regras de produção (se condição então ação). Este conhecimento é avaliado através da correta aplicação, ou não, de cada regra. Uma vez que o estudante atinja 95\% probabilidade de conhecimento de uma regra, ela é considerada aprendida (mastered). Posteriormente, este foi convertido em uma RDB. Há, também, estudos que visam individualizar a inferência deste modelo, de modo a torná-lo mais condizente com as caraterísticas individuais de cada aluno[Yudelson et al. 2013].

Os trabalhos de [Millán et al. 2013] e [Nouh et al. 2006] apresentam estruturas de RBs voltadas para a avaliação do conhecimento do aluno em equações de $1^{\mathrm{o}}$ grau e programação, respectivamente. Estes trabalhos estruturam o conhecimento, de cada domínio, através de agregação de conceitos, ou seja, pequenos conceitos influenciam no conhecimento de um tópico mais abrangente. Por exemplo, no trabalho de [Nouh et al. 2006], um dos tópicos tratados são as Listas Encadeadas;para cada tipo de 
lista há nodos representando as respectivas operações de inserção e remoção de elementos.Ambos os trabalhos utilizam a Teoria de Resposta ao Item, para o cálculo das probabilidades dos nodos que representam os itens de avaliação (questões). Estes nodos são aqueles que recebem as evidências em cada modelo.

Em [Manske and Conati 2005], o componente de avaliação do conhecimento do aluno do software educacional Prime Climb é descrito. Este software utiliza uma RBD para inferir o conhecimento do aluno sobre fatoração de números inteiros, na qual cada time-slice representa uma ação do aluno na tarefa. O trabalho utiliza nodos para representar o fato do aluno conhecer o conceito "Fator Comum", e a fatoração de um número $x$. Também possui um nodo para receber a evidência das ações do estudante, e um para representar o conhecimento anterior.Conforme é relatado em [Manske and Conati 2005], os valores das parametrizações foram obtidos através de análise estatística de prés e póstestes aplicados a 52 alunos.

Em [Seffrin et al. 2013], foi proposta uma RB para avaliar o conhecimento algébrico do estudante, que modela apenas as relações entre as operações de $1^{\circ}$ grau. A modelagem se baseia no princípio de pré-requisitos, ou seja, quais operações devem ser aprendidas primeiramente para que se possa aprender as seguintes. Por exemplo, a propriedade distributiva possui como pré-requisitos a operação de soma e de multiplicação, isto porque ambas são necessárias para a correta aplicação desta propriedade. Este trabalho utiliza como evidência a operação algébrica aplicada pelo aluno nos passos das equações, contidas nos itens de avaliação. Porém, exige que cada equação, e seus respectivos passos, sejam inseridos manualmente na rede.

O modelo proposto neste artigo utiliza uma rede Bayesiana Dinâmica para inferir o conhecimento algébrico do aluno, em operações de $1^{\circ}$ grau. Neste trabalho, pretende-se estender o trabalho proposto em [Seffrin et al. 2013] para utilizar RBDs, e incluir a parte conceitual do domínio algébrico, como igualdades e incógnitas, bem como as principais falsas concepções dos alunos. Tais características não são empregadas nos trabalhos de [Nouh et al. 2006, Millán et al. 2013], uma vez que, nestes trabalhos, são empregadas apenas as operações, suas propriedades e as principais falsas concepções; ou é utilizado somente os conceitos algébricos. Além disso, por utilizarem como evidência os itens de avaliação, é necessário definir manualmente cada um deles na rede, o que, neste trabalho, será evitado com o uso da RBD, já que cada time-slice representará a aplicação (in)correta de uma operação (ou várias) em um passo. Quanto aos outros trabalhos, o Knowledge Tracing não apresenta relações entre os conhecimentos e o modelo do Prime Climb é aplicado a um modelo relativamente pequeno.

\section{Trabalho Proposto}

Como explicado anteriormente, este trabalho é uma extensão do trabalho apresentado em [Seffrin et al. 2013], que definiu uma rede Bayesiana para avaliar o conhecimento algébrico do aluno em equações de $1^{\circ}$ grau. Por possuir a limitação que exigia a definição manual de cada passo de cada equação (o que tornava a rede dependente de cada exercício), este modelo foi portado para uma RBD [Seffrin et al. 2014], o que acarretou uma perda de acurácia em relação a RB não temporal. Com o objetivo de melhorar a acurácia da RBD proposta, foram realizadas entrevistas com dois professores do programa de pós-graduação em computação, de uma universidade no sul do país. Estas entrevistas 
visaram verificar a estrutura da rede e as relações entre as operações de $1^{\mathrm{o}}$ grau.

As entrevistas com professores revelaram que apenas as operações de $1^{\circ}$ grau são insuficientes para inferir o conhecimento do aluno. Isso porque há certos conceitos algébricos por trás das mesmas, tais como a "igualdades entre os termos da equação" que serve de base para o procedimento que pode ser simplificado pela "operação inversa", popularmente conhecido como "passar para o outro lado".

Desta forma, este trabalho propõe uma reestruturação na RBD, combinando os conceitos algébricos, com as suas respectivas operações e falsas concepções. A Figura 1 ilustra a estrutura desta rede, que abrange as operações algébricas de $1^{\circ}$ grau, bem como os conceitos e operações matemáticas utilizados na resolução de tais equações. Esta rede foi modelada utilizando uma relação de pré-requisitos, ou seja, o que se deve conhecer primeiramente para que se possa compreender determinado conteúdo mais complexo. Desta forma, a ordem dos assuntos está de acordo com o que é ensinado em sala de aula. Por exemplo, as operações mais simples, como as 4 operações básicas, se localizam no início da rede. O conteúdo segue até atingir as equações de $1^{\circ}$ grau. Esta modelagem contou com o auxílio de uma especialista, que é professora de matemática e Mestre em Ciências e Matemática.

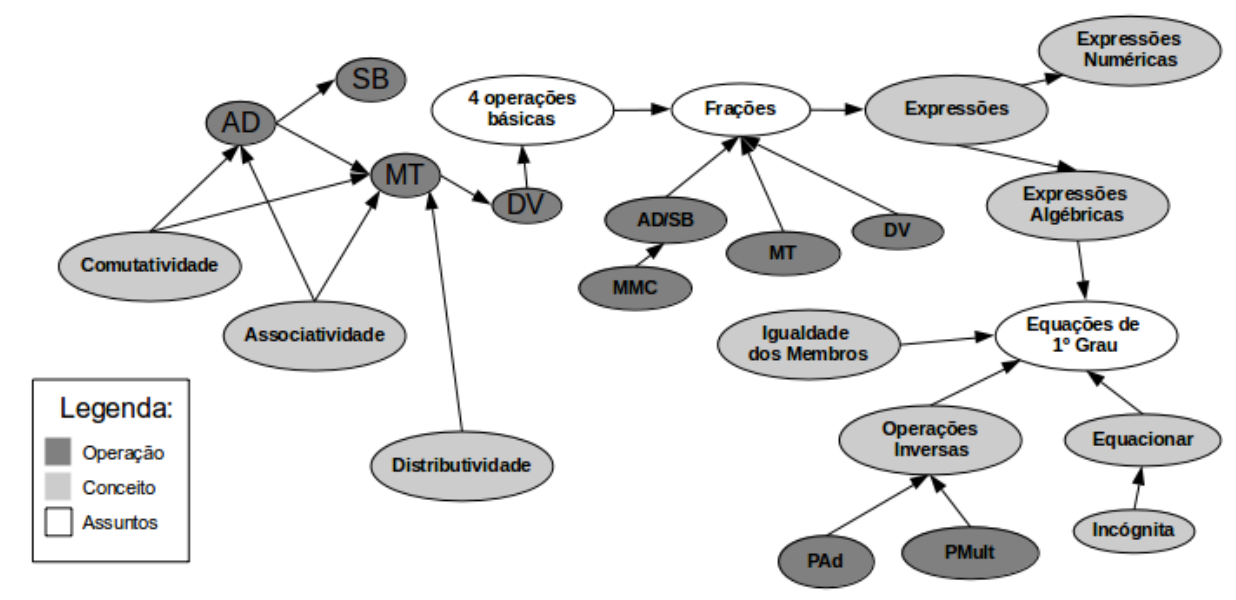

Figura 1. Rede Proposta

No que diz respeito aos assuntos, estes são nodos que agregam as operações com os seus respectivos conceitos e propriedades. Nesta rede estão representados três assuntos: "4 operações básicas", "Frações" e "Equações de $1^{\circ}$ grau".

Observa-se nesta rede a presença de um conjunto de operações de soma, subtração, multiplicação e divisão, específicas para as frações. Isto por que tais operações são aplicadas sobre as frações de forma diferente da forma como são aplicadas aos números inteiros. Por exemplo, para a soma e subtração de frações (nodo "AD/SB") é necessário o conhecimento prévio do cálculo do mínimo múltiplo comum (MMC). Este, por sua vez, exige o conhecimento das operações de multiplicação e divisão. Tal informação é obtida pela relação "4 operações básicas" $\Rightarrow$ "Frações", uma vez que todas as operações aplicadas sobre as frações exigem este conhecimento prévio.

Esta rede contém 2 tipos de nodos-sensores: (i) a (in)correta aplicação de um conhecimento algébrico para resolver uma etapa de uma equação; e (ii) a ocorrência de 
uma falsa concepção. Uma vez incorporado ao STI PAT2Math, tais informações serão obtidas através do componente cognitivo do mesmo, ou seja, o sistema especialista.

\subsection{Cenário}

De modo a ilustrar o funcionamento da rede considere a equação $5 x=50$. Um aluno, ao resolvê-la, submete ao tutor, como próximo passo, a equação $x=\frac{50}{5}$ como solução para esta etapa. O sistema especialista identifica que o estudante aplicou corretamente a habilidade relacionada ao princípio multiplicativo (PMult). Então, no time-slice $t_{0}$ da rede, o nodo-sensor "Passo_Mult" é atualizado, indicando etapa correta. O aluno recebe um feedback positivo do sistema e ele passa à etapa seguinte. Ele encaminha a solução da nova etapa, ou seja, $x=10$, que é identificada como a operação de simplificação (SP), e o nodo-sensor correspondente da RBD é atualizado. Devido à característica temporal da rede, a informação de $t_{0}$ é encaminhada a $t_{1}$. Como a resolução chegou ao fim, o resultado da inferência sobre o conhecimento do estudante é obtido em $t_{1}$, sendo que os nodos da RBD representando as habilidades e conceitos algébricos conterão um percentual representando o domínio do aluno naquela unidade de conhecimento.

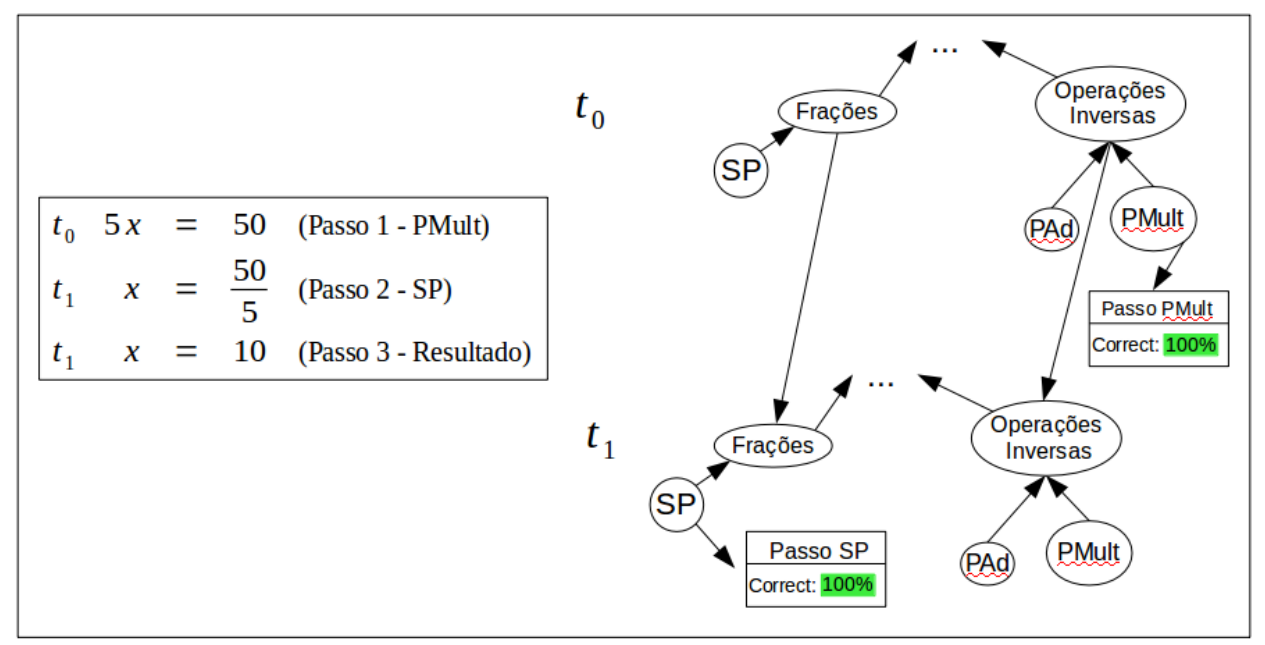

Figura 2. Cenário de execução da rede

\section{Avaliação}

De modo a verificar a estrutura da rede, visando uma correta relação entre os termos e as operações, foram conduzidas entrevistas com três professores de matemática, todos com mais de 10 anos de docência. Dois professores atuam no ensino superior e o outro é professor do ensino fundamental. As entrevistas ocorreram da seguinte forma. Inicialmente, o entrevistado era contextualizado sobre o uso da estrutura para mapear o conhecimento do aluno e a necessidade de estruturar do conhecimento algébrico. Em seguida, esta estrutura foi analisada por cada um dos entrevistados. Cada um emitiu o seu parecer sobre ela, comentando a adição de alguns nodos extras, representando conceitos não expressos na rede. Algumas sugestões foram a inserção de nós referentes à operação entre os sinais + e -, a propriedade algébrica por trás da operação inversa, e a precedência de operações. Cada entrevista ocorreu de forma individual, não havendo contato entre os entrevistados. 
Como resultado da avaliação, os entrevistados concordaram com a estrutura geral da rede, e sugeriram alguns conceitos extras que poderiam ser incluídos, tais como (Figura $3)$ :

- Interpratação e Abstração: Nodos relacionados ao conceito de "equacionar", ou seja, obter uma equação a partir de uma situação-problema. Estas são duas habilidades necessárias ao aluno para a obtenção de tal equação: interpretar o texto e saber abstrair determinados termos em expressões com incógnitas.

- Organizar: Se refere à reorganização dos termos da equação de modo a calcular o resultado da incógnita, isolando os números inteiros em um lado da equação e os valores com incógnita no lado oposto. Por exemplo, na equação $3 x+4=2 x+5$, pode ser reorganizada em $3 x-2 x=5-4$, cuja resolução é $x=1$.

- Sinais: Nodo que representa as operações envolvendo os sinais dos termos, como: $-\times-=+,+\times-=-,-\times+=-\mathrm{e}+\times+=+$.

- Propriedade algébrica: Foi apontado a necessidade de um nodo que represente a propriedade da álgebra, na qual toda a operação aplicada em um lado da equação, deve ser aplicada também no lado oposto, de modo a manter o equilíbrio da equação.

- Ordem de prioridade dos operadores: Se refere à ordem de aplicação das 4 operações fundamentais. Na matemática, as operações de divisão e multiplicação possuem prioridade sobre as operações de soma e subtração, e, portanto, devem ser aplicadas primeiramente.

- Simplificação: A operação de simplificação de frações, na qual o numerador e o denominador de uma fração são divididos por um mesmo número inteiro, até que a mesma se torne irredutível.

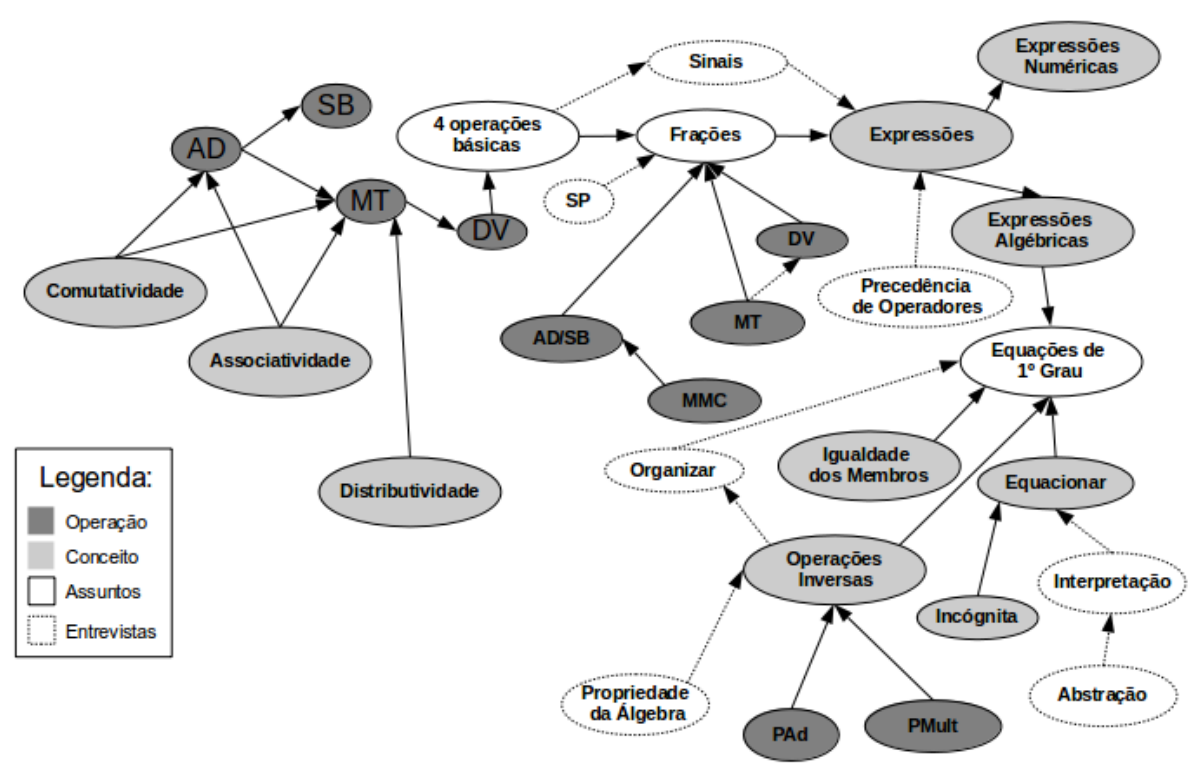

Figura 3. Rede Proposta com as alterações sugeridas pelos entrevistados

Visando a implementação das falsas concepções na rede, foi questionado sobre as principais dificuldades que os alunos apresentam e que os entrevistados observam no cotidiano de sala de aula. A principal dificuldade apontada, pelos três, foi a que envolve 
as operações inversas, ou o popular "passa para o outro lado". É observada a dificuldade da compreensão, por parte de alguns estudantes, sobre os conceitos por trás deste método de cálculo. Alguns exemplos de erros apontados pelos entrevistados, envolvendo este método são:

$$
\begin{aligned}
3 x=2 & \Rightarrow x=3 \times 2 \\
x=\frac{2 x}{2}+4 & \Rightarrow 2 x=2 x+4 \\
5=2 x & \Rightarrow \frac{5}{-2}=x
\end{aligned}
$$

Conforme ilustrado pela ultima equação desta figura, há também a dificuldade de compreender a diferença entre operação e sinal, o que resulta no estudante, ao trocar um termo de lado, acabar trocando também o seu sinal.

Outros erros apontados envolveram a operação de simplificação de termos em frações, como $\frac{x+2}{2}=3 \rightarrow x=3$, e a aplicação da propriedade distributiva em situações indevidas, como $2 \times 3 \times x \rightarrow(2 \times 3) \times(2 x) \rightarrow 12 x$. Também foi apontada a dificuldade alunos do ensino fundamental de lidar com as 4 operações básicas.

\section{Conclusão}

A capacidade de mapear o conhecimento do aluno permite aos Sistemas Tutores Inteligentes serem mais adaptativos na assistência provida. Uma das técnicas utilizadas para modelagem do conhecimento do aprendiz são as redes Bayesianas, que são estruturas de dados capazes de lidar com incertezas. Embora, as RB já tivessem sido utilizadas por outros trabalhos para representar o conhecimento algébrico do estudantes, esses trabalhos relacionados apresentavam duas principais lacunas. As redes eram dependentes dos exercícios, ou seja, existia um nó de evidência para cada exercício resolvido pelo aluno. Isso acarretava complexidade à rede, assim como restringia o número de exercícios que o STI poderia aplicar. Uma outra lacuna observada era que essas redes modelavam apenas as habilidades dos alunos, ou seja, o conhecimento dos estudantes sobre as operações algébricas. Porém, igualmente importante é a modelagem dos conceitos algébricos e suas relações com as operações.

Deste modo, neste artigo foi apresentada uma estrutura de Rede Bayesiana Dinâmica que combina as operações de $1^{\circ}$ grau, com os seus respectivos conceitos e propriedades. A rede também considera como evidência a operação aplicada pelo aluno em cada passo, se tornando independente de domínio.

De modo a validar a estrutura desta rede foram realizadas entrevistas com três professores de matemática. Tais entrevistas visaram verificar a relação entre as operações e os conceitos, e levantar os principais erros cometidos pelos alunos. Estas entrevistas mostraram que a estrutura proposta é válida para representar as relações entre os conceitos e operações de equações de $1^{\circ}$ grau. Também foi constatado que as maiores dificuldades dos estudantes estão na compreensão dos conceitos relacionados à operação inversa, operação entre sinais, simplificação de termos na fração e a propriedade distributiva.

Como trabalhos futuros pretende-se incluir as principais falsas concepções algébricas, obtidas através das entrevistas, na estrutura proposta. Através de dados de resolução de equações, por parte de alunos de $6^{\mathrm{a}}$ e $7^{\mathrm{a}}$ série, pretende-se definir as probabilidades da rede. Uma vez que estas probabilidades forem definidas, esta rede será submetida a uma avaliação utilizando alunos simulados, de modo a verificar a capacida- 
des preditiva da rede. Finalmente, uma vez que a rede esteja validada pela simulação, a rede será incorporada ao STI PAT2Math para ser avaliada com alunos reais.

\section{Agradecimentos}

O presente trabalho foi realizado com o apoio dos seguintes órgãos de fomento à pesquisa: CNPq e FAPERGS.

\section{Referências}

Corbett, A. and Anderson, J. (1994). Knowledge tracing: Modeling the acquisition of procedural knowledge. User modeling and user-adapted interaction, 4(4):253-278.

Duijnhoven, J. V. (2003). Knowledge assessment using bayesian networks: A case study in the domain of algebraic expectation. Master's thesis cognitive artificial intelligence, Utrecht University, Utrecht.

Manske, M. and Conati, C. (2005). Modelling Learning in an Educational Game. In Proceedings of the 2005 conference on Artificial Intelligence in Education: Supporting Learning through Intelligent and Socially Informed Technology, pages 411-418, Amsterdam. IOS Press.

Matz, M. (1982). Towards a process model for high school algebra errors. In Sleeman, D. and Brown, J. S., editors, Intelligent Tutoring Systems, Computers and People, chapter 2, pages 25-50. Academic Press, London, UK.

Millán, E., Descalço, L., Castillo, G., Oliveira, P., and Diogo, S. (2013). Using Bayesian networks to improve knowledge assessment. Computers \& Education, 60(1):436-447.

Nouh, Y., Karthikeyani, P., and Nadarajan, R. (2006). Intelligent tutoring system-bayesian student model. In Proceedings of 1st IEEE International Conference on Digital Information Management (ICDIM), pages 257-262, Bangalore, India. IEEE.

Pearl, J. (1988). Probabilistic Reasoning in Intelligent Systems: Networks of Plausible Inference. Morgan Kaufmann, San Francisco, CA.

Russell, S. J. and Norvig, P. (2002). Artificial Intelligence: A Modern Approach. Prentice Hall, Upper Saddle River, NJ, second edition.

Seffrin, H., Rubi, G., and Jaques, P. (2013). Uma rede bayesiana aplicada à modelagem do conhecimento algébrico do aprendiz. In Anais do Simpósio Brasileiro de Informática na Educação, Campinas, SP. Sociedade Brasileira de Computação.

Seffrin, H., Rubi, G., and Jaques, P. (2014). A dynamic bayesian network for inference of learners algebraic knowledge. In Proceedings of the 29th Symposium On Applied Computing, Gyeongju, Korea. ACM Press.

Vanlehn, K. (2006). The Behavior of Tutoring Systems. International Journal of Artificial Intelligence in Education, 16(3):227-265.

Woolf, B. P. (2008). Building intelligent interactive tutors, volume 3. Morgan Kaufmann, San Francisco, CA.

Yudelson, M. V., Koedinger, K., and Gordon, G. J. (2013). Individualized Bayesian Knowledge Tracing Models. In Proceedings of Artificial Intelligence in Education, volume 7926 of Lecture Notes in Computer Science, pages 171-180, Memphis, TN. Springer Verlag. 